\title{
Dark Matter implications of Fermi-LAT measurement of anisotropies in the diffuse gamma-ray background
}

\author{
G.A. Gómez-Vargas \\ Universidad Autónoma de Madrid \& Instituto de Física Teórica UAM/CSIC, Cantoblanco, E-28049 Madrid, \\ Spain and \\ Istituto Nazionale di Fisica Nucleare, Sez. Roma Tor Vergata, Roma, Italy \\ A. Cuoco \\ The Oskar Klein Centre for Cosmo Particle Physics, AlbaNova, SE-106 91 Stockholm, Sweden \\ T. Linden \\ Department of Physics, University of California, Santa Cruz, CA 95064, USA \\ M.A. Sánchez-Conde \\ SLAC National Accelerator Laboratory \& Kavli Institute for Particle Astrophysics and Cosmology, Menlo Park, \\ CA 94025, USA \\ J.M. Siegal-Gaskins \\ California Institute of Technology, Pasadena, CA 91125, USA
}

\section{For the Fermi-LAT Collaboration \\ and}

T. Delahaye

LAPTh, Universit e de Savoie, CNRS, 9 chemin de Bellevue, BP110, F-74941 Annecy-le-Vieux Cedex, France

Institut d'Astrophysique de Paris, UMR 7095 - CNRS, Universit e Pierre \& Marie Curie, 98 bis boulevard Arago, 75014, Paris, France and

Instituto de Física Teórica UAM/CSIC, Cantoblanco, E-28049 Madrid, Spain

M. Fornasa

School of Physics and Astronomy, University of Nottingham, NG7 2RD Nottingham, United Kingdom

E. Komatsu

Max-Planck-Institut fur Astrophysik, Karl-Schwarzschild Str. 1, 85741 Garching, Germany

Kavli Institute for the Physics and Mathematics of the Universe, Todai Institutes for Advanced Study,

the University of Tokyo, Kashiwa, Japan 277-8583 (Kavli IPMU, WPI) and

Texas Cosmology Center and the Department of Astronomy, The University of Texas at Austin, 1 University Station, C1400, Austin, TX 78712, USA

F. Prada

Campus of International Excellence UAM+CSIC, Cantoblanco, E-28049 Madrid, Spain

Instituto de Física Teórica UAM/CSIC, Cantoblanco, E-28049 Madrid, Spain and

Instituto de Astrofísica de Andalucía (CSIC), Glorieta de la Astronomía, E-18080 Granada, Spain

J. Zavala

Perimeter Institute for Theoretical Physics, 31 Caroline St. N., Waterloo, ON, N2L 2Y5, Canada and

Department of Physics and Astronomy, University of Waterloo, Waterloo, Ontario, N2L 3G1, Canada

\begin{abstract}
The detailed origin of the diffuse gamma-ray background is still unknown. However, the contribution of unresolved sources is expected to induce small-scale anisotropies in this emission, which may provide a way to identify and constrain the properties of its contributors. Recent studies have predicted the contributions to the angular power spectrum (APS) from extragalactic and galactic dark matter (DM) annihilation or decay. The Fermi-LAT collaboration reported detection of angular power with a significance larger than $3 \sigma$ in the energy range from $1 \mathrm{GeV}$ to 10 $\mathrm{GeV}$ on 22 months of data Ackermann et al. 2012. For these preliminary results the already published Fermi-LAT APS measurements Ackermann et al. 2012 are compared to the accurate predictions for DM anisotropies from state-of-the-art cosmological simulations as presented in Fornasa et al. 2013] to derive constraints on different DM candidates.
\end{abstract}

\section{THE DIFFUSE GAMMA-RAY BACKGROUND}

The diffuse gamma-ray background is characterized by an isotropic or nearly isotropic distribution and is therefore known as the Isotropic Gamma-ray Background (IGRB) Kraushaar et al. 1972. It is constituted by gamma rays produced by various sources, including blazars, pulsars, and possible DM structures, not yet detected due to the limited angular resolution and photon statistics of the Fermi-LAT. Figure 1 shows the IGRB spectrum and the estimated contributions from unresolved blazars, star-forming and radio galaxies. The angular distribution of photons in the diffuse background may contain information about the presence and the nature of these unresolved source populations. 


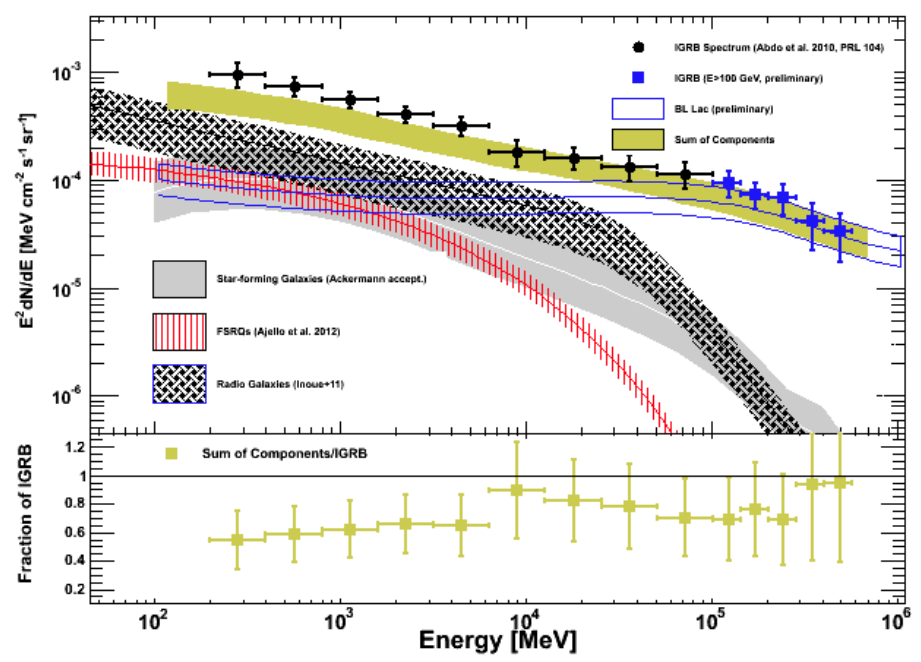

Figure 1: IGRB spectrum and the contribution from all the different extragalactic source classes that have been detected by Fermi. The golden band shows the sum of all the source populations. By summing all contributions it is apparent that there is still room for other components at all energies within the uncertainties Sanchez-Conde 2012]. See also [Stecker and Venters 2011. for other theoretical estimates of the relative contributions of unresolved blazars and star-forming galaxies to the IGRB.

\section{FERMI-LAT MEASUREMENT OF THE ANGULAR POWER SPECTRUM IN THE IGRB}

In Ackermann et al. 2012 the first 22 months of Fermi-LAT data were analyzed, dividing the energy range between $1 \mathrm{GeV}$ and $50 \mathrm{GeV}$ in 4 energy bins. The point sources in the first year catalogue Abdo et al. 2010 have been masked, as well as the emission within a band of 30 degrees above and below the Galactic plane . The masking was done to cover the regions in the sky where the emission is dominated by resolved sources and by the Galactic foreground, and to restrict the analysis only to where the IGRB is a significant component.

Two definitions for anisotropies were used in Ackermann et al. 2012:

- Intensity APS: An intensity map $I(\psi)$ can be decomposed in spherical harmonics,

$$
I(\psi)=\sum_{l m} a_{l m} Y_{l m}(\psi),
$$

where coefficients $a_{l m}$ determine the APS which is given by $C_{l}=\left\langle\left|a_{l m}\right|^{2}\right\rangle$. This definition is particularly useful because it gives us the dimensionful size of intensity fluctuations and can be compared with predictions for source classes whose collective intensity is known or assumed.

- Fluctuation APS: can be derived from the intensity APS, dividing by the average intensity squared. The fluctuation APS is energyindependent for a single source class, if all mem- bers of the source class share the same observed energy spectrum.

The Fermi-LAT collaboration reported detection of angular power in all 4 energy bins considered, with a signicance larger than $3 \sigma$ in the energy bins from $1 \mathrm{GeV}$ to $10 \mathrm{GeV}$. The data have been compared with the APS of a source model made of i) the point sources in Abdo et al. 2010, ii) a model for the interstellar diffuse emission and iii) an isotropic component at the level of the IGRB in Abdo et al. 2010. The model angular power at $155 \leq l \leq 504$ is consistently below that measured in the data.

Despite the mask applied along the Galactic plane, some known Galactic emission can extend to high latitudes. Therefore a model of the Galactic foregrounds was subtracted from the data, and then the APS of the residual maps was calculated. This measurement is referred to as the cleaned data in Ackermann et al. 2012]. We use this second measurement in this work.

\section{DARK MATTER PREDICTIONS}

The APS of gamma rays from DM annihilations or decays has been computed from the all-sky template maps produced in Fornasa et al. 2013]. The authors of Fornasa et al. 2013 used the Millennium-II N-body simulation to model the abundance and the clustering of extragalactic DM halos and subhalos. The technique presented in Zavala et al. 2010, based on the random repetition of copies of the Millennium-II simulation box, is implemented to probe the universe up to $z=2$. The emission from DM halos with a mass below the resolu- 
tion of the simulation was estimated assuming that the halo number density and the mass-luminosity relation obtained from the halos in Millennium-II remains unchanged below the resolution, down to the minimal selfbound halo mass $M_{\min }$. On the other hand, the contribution of low mass subhalos was modeled following the technique described in Kamionkowski et al. 2010 and extended in [Sánchez-Conde et al. 2011].

The smooth DM halo of the Milky Way was parametrized in Fornasa et al. 2013 as an Einasto profile, since this provides the best fit to the Milky Waylike halo obtained in the Aquarius N-body simulation. Galactic subhalos down to $10^{5} M_{\odot}$ were accounted for directly from the Aquarius simulation, while we use the same procedure as before to account for the contribution of unresolved subhalos. It has been shown that such objects do not contribute significantly to the total intensity APS.

In Fornasa et al. 2013 the effect of the assumptions made in the modeling of the DM distribution also was estimated, looking for their effect both on the intensity of the DM-induced emission and on its APS. The two most relevant sources of uncertainty are the amount of substructures hosted by DM halos and the value of $M_{\min }$. The first gives rise to an uncertainty of a factor 20-30 both in the average intensity and in the intensity APS; when the uncertainty on the value of $M_{\min }$ is taken into account, these factors build up to 40 and 100 for the average intensity and the intensity APS, respectively.

\section{SETTING CONSTRAINTS}

Here we present the method used to set conservative limits on the thermally averaged cross section, $\langle\sigma v\rangle$, for DM annihilation into three different channels, $b \bar{b}$ quarks, $\mu^{+} \mu^{-}$, and $\tau^{+} \tau^{-}$leptons.

- To set constraints we use the foreground-cleaned $C_{p}$ shown in table II of Ackermann et al. 2012. There are four $C_{p}$ values measured corresponding to four energy bins, $1-2 \mathrm{GeV}, 2-5 \mathrm{GeV}, 5-10$ $\mathrm{GeV}$, and $10-50 \mathrm{GeV}$. We use them independently to set limits.

- Requiring that the DM-induced intensity APS averaged in $155<l<204$ does not overshoot the measured $C_{p}$ in the $155<l<504$ multipole range plus 1.64 times its error leads to $95 \%$ CL limits on $\langle\sigma v\rangle^{1}$.

\footnotetext{
${ }^{1}$ We assume Gaussian erros, then the value of 1.64 is based on the fact that $95 \%$ of the area of a Gaussian distribution is within 1.64 standard deviations of the mean.
}

- We know that the IGRB anisotropy has multiple contributors, therefore these constraints are conservative. Other contributors to IGRB anisotropy are not well known, but we already have constraints on the contribution of blazars Cuoco et al. 2012. We subtract this contribution from the measured $C_{p}$ and require that the DM-induced APS does not overshoot this new limit.

\section{PRELIMINARY RESULTS}

Figures 2- 4 show the 95\% CL limits on the annihilation cross section for three different channels, $b \bar{b}$ quarks, $\mu^{+} \mu^{-}$, and $\tau^{+} \tau^{-}$leptons, respectively. The main uncertainty in the predictions obtained in Fornasa et al. 2013 lies in the properties of low-mass subhalos, below the mass resolution of the simulations. Different values of the "subhalo boost" strongly affect the prediction for the DM-induced gamma-ray intensity and its anisotropies. Fornasa et al. 2013 considered two benchmark scenarios for subhalos, assuming that the uncertainties can be modeled by changing the subhalo abundance: i) the LOW case, where halos are relatively poor in subhalos, according to the predictions of Kamionkowski et al. 2010 and Sánchez-Conde et al. 2011; the constraints using this scenario are shown in the left-upper panel of figures 2-4, and ii) the HIGH case, with large subhalo boosts, compatible with what was found by Pinzke et al. 2011, Gao et al. 2012]; the right-upper and lower panels in the figures 2 - 4 use this case. In the lower panels the predictions for the DM-induced APS (with a HIGH subhalo boost) are compared with the APS measured by Fermi-LAT once the contribution from blazars is subtracted. This represents the case where the most stringent constraints on $\langle\sigma v\rangle$ are obtained. We are currently updating the Fermi-LAT APS measurement using $\sim 45$ months of data, we expect to improve the constraints presented in these proceedings after include the new measurement in the method shown in section 4 . 


\section{Acknowledgments}

This work was partially supported by the Spanish MINECO's Consolider-Ingenio 2010 Programme under grant MultiDark CSD2009-00064.

The work of GAGV was supported in part by MINECO under grants FPA2009-08958, FPA200909017 and FPA2012-34694, and under the 'Centro de Excelencia Severo Ochoa' Programme SEV-2012-0249, by the Comunidad de Madrid under grant HEPHACOS S2009/ESP-1473, and by the European Union under the Marie Curie-ITN program PITN-GA-2009-237920.

GAGV thanks Caltech for hospitality during the completion of this work.

The work of TD was supported in part by the ANR project DMAstroLHC, ANR-12-BS05-0006-01.

JZ is supported by the University of Waterloo and the Perimeter Institute for Theoretical Physics. Research at Perimeter Institute is supported by the Government of Canada through Industry Canada and by the Province of Ontario through the Ministry of Research \& Innovation. JZ acknowledges financial support by a CITA National Fellowship.

The Fermi LAT Collaboration acknowledges support from a number of agencies and institutes for both development and the operation of the LAT as well as scientific data analysis. These include NASA and DOE in the United States, CEA/Irfu and IN2P3/CNRS in France, ASI and INFN in Italy, MEXT, KEK, and JAXA in Japan, and the K. A. Wallenberg Foundation, the Swedish Research Council and the National Space Board in Sweden. Additional support from INAF in Italy and CNES in France for science analysis during the operations phase is also gratefully acknowledged.

\section{References}

Fornasa, M., Zavala, J., Sánchez-Conde, M. A., SiegalGaskins, J. M., Delahaye, T., Prada, F., Vogelsberger, M., Zandanel, F., Frenk, C. S. 2013. Characterization of dark-matter-induced anisotropies in the diffuse gamma-ray background. Monthly Notices of the Royal Astronomical Society 429, 1529-1553.

Ackermann, M., and 136 colleagues 2012. Anisotropies in the diffuse gamma-ray background measured by the Fermi LAT. Physical Review D 85, 083007.

Kraushaar, W. L., Clark, G. W., Garmire, G. P., Borken, R., Higbie, P., Leong, V., Thorsos, T. 1972. High-Energy Cosmic Gamma-Ray Observations from the OSO-3 Satellite. The Astrophysical Journal 177, 341.
Sanchez-Conde, M. A. 2012. Dark matter constraints from the Fermi/LAT Extragalactic Gamma-ray Background and the role of halo substructure. American Astronomical Society Meeting Abstracts \#220 220, \#116.05.

Stecker, F. W., Venters, T. M. 2011. Components of the Extragalactic Gamma-ray Background. The Astrophysical Journal 736, 40.

Abdo, A. A., and 235 colleagues 2010. Fermi Large Area Telescope First Source Catalog. The Astrophysical Journal Supplement Series 188, 405-436.

Abdo, A. A., and 183 colleagues 2010. Spectrum of the Isotropic Diffuse Gamma-Ray Emission Derived from First-Year Fermi Large Area Telescope Data. Physical Review Letters 104, 101101.

Zavala, J., Springel, V., Boylan-Kolchin, M. 2010. Extragalactic gamma-ray background radiation from dark matter annihilation. Monthly Notices of the Royal Astronomical Society 405, 593-612.

Kamionkowski, M., Koushiappas, S. M., Kuhlen, M. 2010. Galactic substructure and dark-matter annihilation in the Milky Way halo. Physical Review D 81, 043532 .

Sánchez-Conde, M. A., Cannoni, M., Zandanel, F., Gómez, M. E., Prada, F. 2011. Dark matter searches with Cherenkov telescopes: nearby dwarf galaxies or local galaxy clusters?. Journal of Cosmology and Astro-Particle Physics 12, 11.

Pinzke, A., Pfrommer, C., Bergström, L. 2011. Prospects of detecting gamma-ray emission from galaxy clusters: Cosmic rays and dark matter annihilations. Physical Review D 84, 123509.

Gao, L., Frenk, C. S., Jenkins, A., Springel, V., White, S. D. M. 2012. Where will supersymmetric dark matter first be seen?. Monthly Notices of the Royal Astronomical Society 419, 1721-1726.

Cuoco, A., Komatsu, E., Siegal-Gaskins, J. M. 2012. Joint anisotropy and source count constraints on the contribution of blazars to the diffuse gamma-ray background. Physical Review D 86, 063004. 

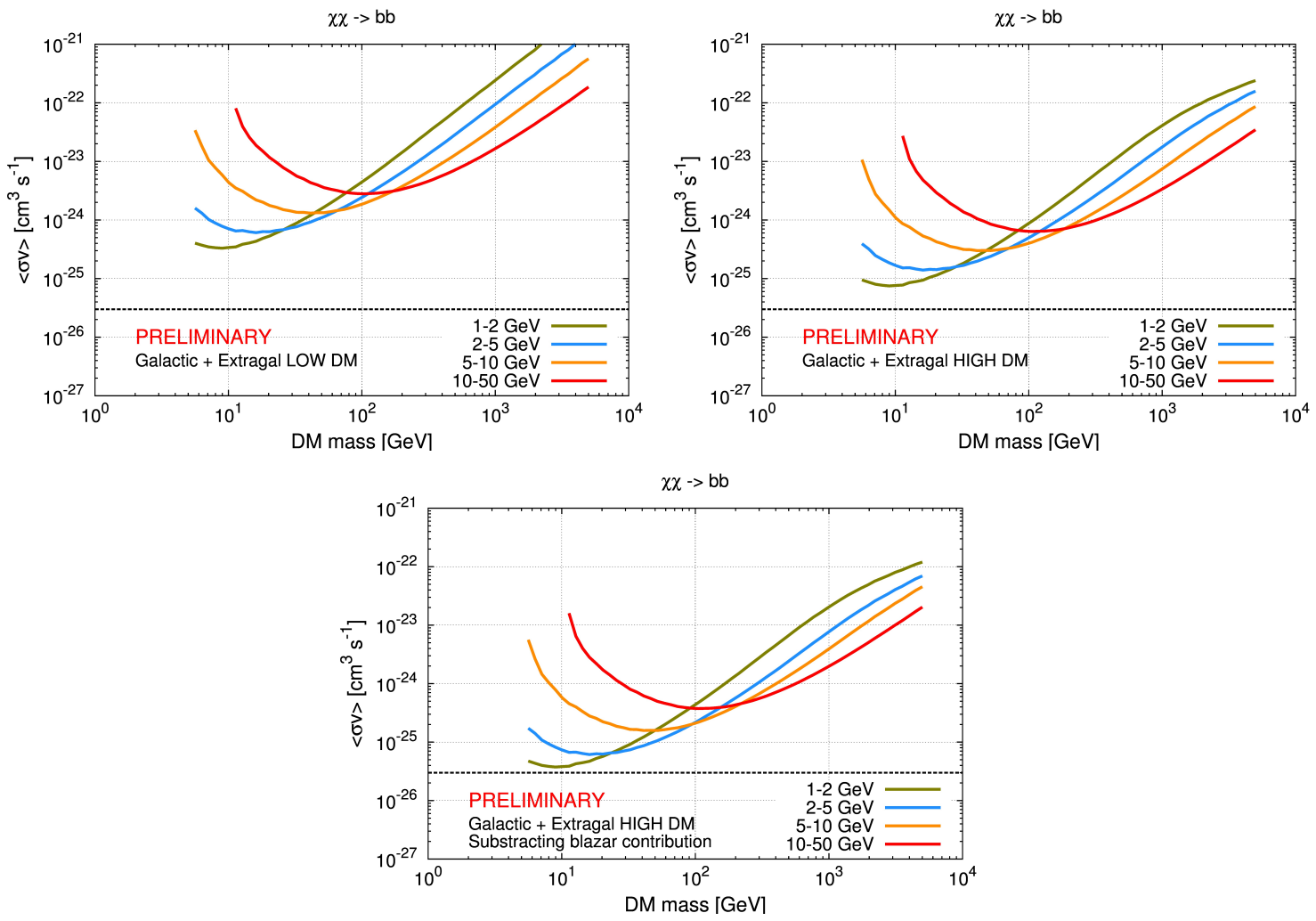

Figure 2: 95\% CL limits on the annihilation cross section for the $b \bar{b}$ channel.
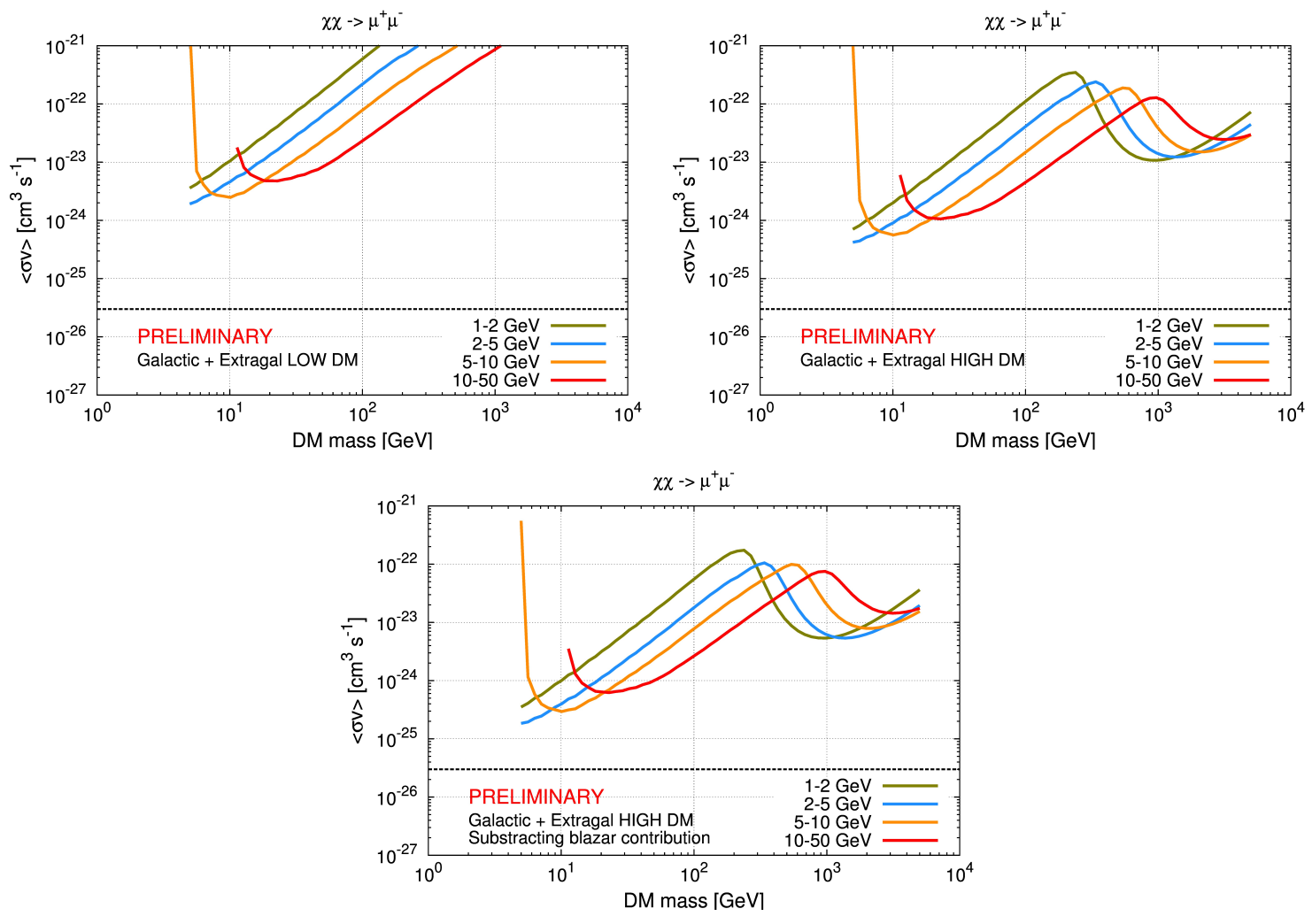

Figure 3: 95\% CL limits on the annihilation cross section for the $\mu^{+} \mu^{-}$channel. 

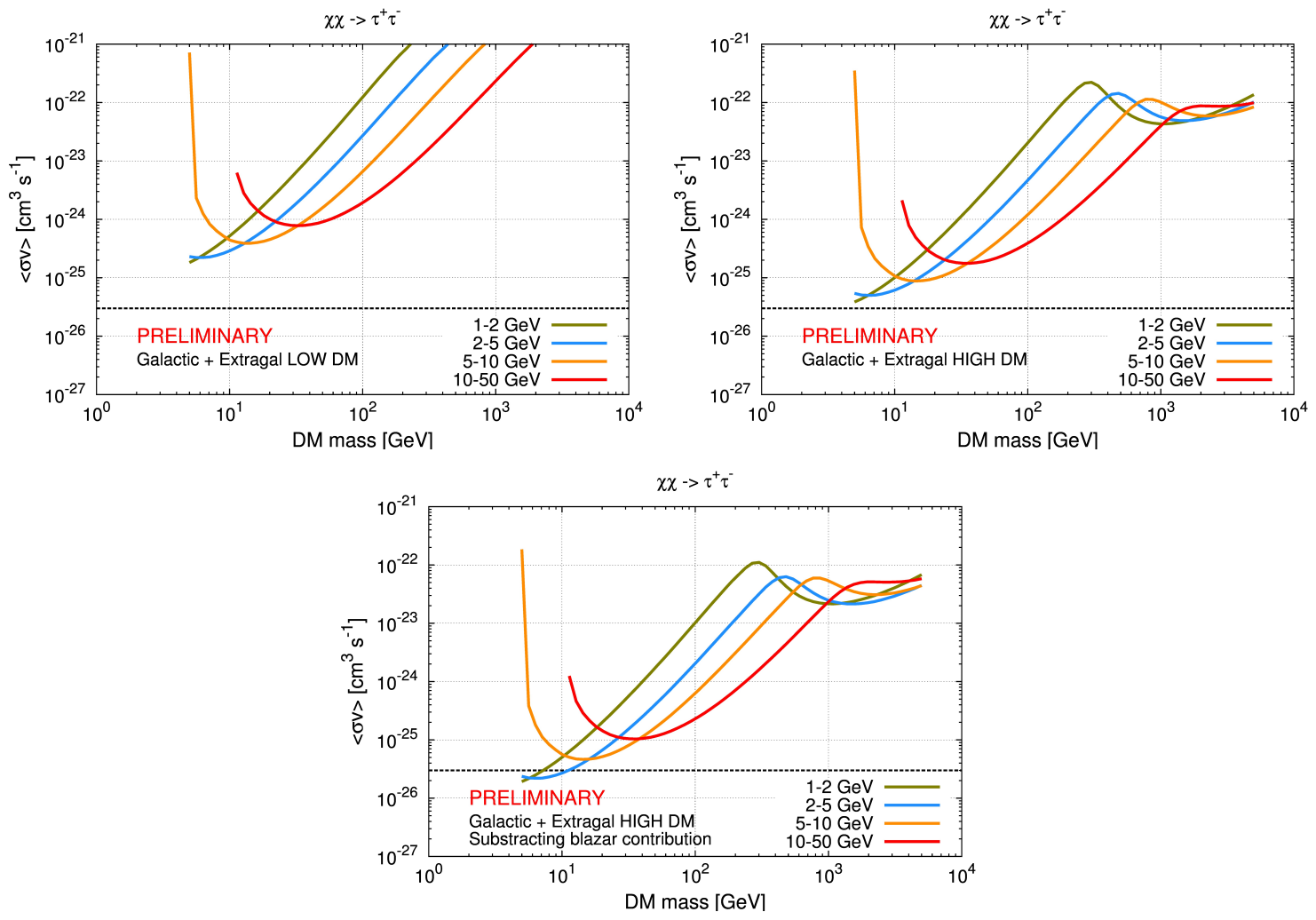

Figure 4: 95\% CL limits on the annihilation cross section for the $\tau^{+} \tau^{-}$channel. 